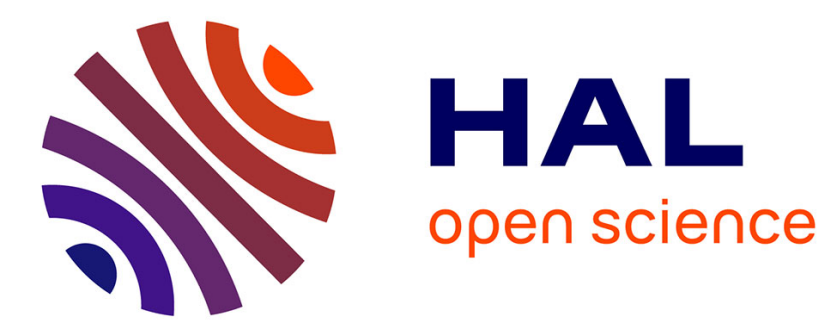

\title{
Biomechanical response of the CNS is associated with frailty in NPH-suspected patients
}

\author{
A. Vallet, N. del Campo, E. Hoogendijk, A. Lokossou, Olivier Balédent, Z. \\ Czosnyka, L. Balardy, P. Payoux, P. Swider, Sylvie Lorthois, et al.
}

\section{- To cite this version:}

A. Vallet, N. del Campo, E. Hoogendijk, A. Lokossou, Olivier Balédent, et al.. Biomechanical response of the CNS is associated with frailty in NPH-suspected patients. Journal of Neurology, 2020, 267 (5), pp.1389-1400. 10.1007/s00415-019-09689-z . hal-03011641

\section{HAL Id: hal-03011641 https://hal.science/hal-03011641}

Submitted on 25 Nov 2020

HAL is a multi-disciplinary open access archive for the deposit and dissemination of scientific research documents, whether they are published or not. The documents may come from teaching and research institutions in France or abroad, or from public or private research centers.
L'archive ouverte pluridisciplinaire HAL, est destinée au dépôt et à la diffusion de documents scientifiques de niveau recherche, publiés ou non, émanant des établissements d'enseignement et de recherche français ou étrangers, des laboratoires publics ou privés. 


\section{OATAO \\ Open Archive Toulouse Archive Ouverte}

\section{Open Archive Toulouse Archive Ouverte}

OATAO is an open access repository that collects the work of Toulouse researchers and makes it freely available over the web where possible

This is an author's version published in: https://oatao.univ-toulouse.fr/25380

\section{Official URL:}

https://doi.org/10.1007/s00415-019-09689-z

\section{To cite this version:}

Vallet, Alexandra $\fallingdotseq$ and Del Campo, Natalia and Hoogendijk, Emiel O. and Lokossou, Armelle and Balédent, Olivier and Czosnyka, Zofla and Balardy, Laurent and Payoux, Pierre and Swider, Pascal Schmidt, Eric Biomechanical response of the CNS is associated with frailty in $\mathrm{NPH}$-suspected patients. (2020) Journal of Neurology, 267. 1389-1400. ISSN 0340-5354.

Any correspondence concerning this service should be sent to the repository administrator: tech-oatao@listes-diff.inp-toulouse.fr 


\title{
Biomechanical response of the CNS is associated with frailty in NPH-suspected patients
}

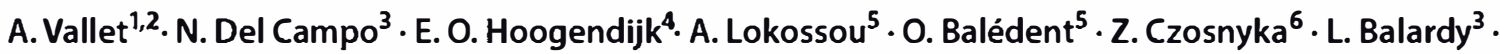 \\ P. Payoux ${ }^{2,3}$. P. Swider ${ }^{1}$. S. Lorthois' ${ }^{1}$ E. Schmidt ${ }^{2,3}$
}

Recelved: 2 September 2019/ Revlsed: 20 December 2019 / Accepted: 23 December 2019

(c) Springer-Verlag GmbH Germany, part of Springer Nature 2020

\begin{abstract}
Frailty is known to predict dementia. However, its link with neurodegenerative alterations of the central nervous system (CNS) is not well understood at present. We investigated the associationbetween the biomechanical response of the CNS and frailty in older adults suspected of normal pressure hydrocephalus (NPH) presenting with markers of multiple co-existing pathologies. The biomechanical response of the CNS was characterized by the CNS elastance coefficient inferred from phase contrast magnetic resonance imaging and intracranial pressure monitoring during a lumbar infusion test. Frailty was assessed with an index of health deficit accumulation. We found a significant association between the CNS elastance coefficient and frailty, with an effect size comparable to that between frailty and age, the latter being the strongest known risk factor for frailty. Results were independent of CSF dynamics, showing that they are not specific to the NPH neuropathological condition. The CNS biomechanical characterization may help to understand how frailty is related to neurodegeneration and detect the shift from normal to pathological brain ageing.
\end{abstract}

Keywords Biomechanics $\cdot$ Frailty $\cdot$ Normal pressure hydrocephalus $\cdot$ Lumbar infusion test $\cdot$ Phase contrast magnetic resonance imaging $\cdot$ Neurodegenerative CNS changes

\section{Introduction}

It is well established that the accumulation of neurodegenerative brain pathologies occurs years before the onset of clinical symptoms [1]. This long preclinical stage represents a unique opportunity for preventive therapeutic strategies

S. Lorthois and E. Schmidt jointly supervised this work.

A. Vallet

alexandra.vallet@imft.fr

E. Schnidt

schmidt.e@chu-toulouse.fr

1 Institut de Mécanique des Fluides de Toulouse (IMFT), Université de Toulouse, CNRS, Toulouse, France

2 ToNIC UMR 1214, INSERM, Toulouse, France

3 Centre Hospitalier Universitaire de Toulouse, Toulouse, France

4 Department of Epidenuiology and Biostatistics, Amsterdam Public Health Research Institute, Amsterdam UMC, VU University Medical Center, Amsterdam, The Netherlands

5 CHIMERE EA 7516, Amiens, France

6 Academic Neurosurgical Unit, Cambridge University Hospital, Cambridge, UK [2]. A major unresolved challenge is the identification of mechanisms that can be quantified before clinical signs become significant.

Frailty is conceived as an acceleration of the ageing process leading to an increased risk of negative health-related outcomes [3] including dementia. Even if it is not brain specific, frailty has been demonstrated to predict Alzheimer's disease $(\mathrm{AD})$ and related dementia [4-6], and has been associated with cognitive impairments [7-9], structural brain abnormalities [10, 11], cortical brain infarcts [10] and subclinical levels of brain neuropathologies [12]. It is thought that frailty and pathological brain ageing share common underlying neurobiological processes. Quantifying these brain-specific processes might open a window of opportunity for the prevention of neurodegenerative diseases. 
The recent application of biomechanical models in neurodegenerative diseases has provided ground-breaking insights into the brain [13], related, for example, to the interstitial fluid transport in the parenchyma [14] or the occlusion of capillaries in $\mathrm{AD}$ [15]. It allows the dynamic response of a system to mechanical stressors to be quantified and therefore provides information on its mechanical resilience.

The objective of the present study was to explore the potential of the biomechanical approach to better characterize the brain-specific processes shared by both brain pathological ageing and frailty. We hypothesized that the biomechanical response of the CNS could be associated with frailty in older patients presenting with neurodegenerative pathologies. This hypothesis was tested on a cohort of patients suspected of normal pressure hydrocephalus (NPH) as (1) these patients often present with co-existing neurodegenerative diseases [16, 17] and (2) the lumbar infusion test, which is performed as part of their clinical assessment [18], is suitable for measuring the biomechanical response of the CNS.

\section{Methods}

\section{Study population}

One hundred patients suspected of NPH (46 women and 54 men aged 52-92 years) were included in the PROLYPHIC cohort between 2013 and 2015 at Toulouse University Hospital. The suspicion of NPH was based on established guidelines [19], i.e. the presence of (1) clinical symptoms of gait or balance disturbance, cognitive impairment or urinary disorders, (2) neuroimaging evidence of ventriculomegaly (i.e. Evans Index > 0.3) and (3) narrow callosal angle, temporal horn enlargement or periventricular signal changes.

\section{Standard protocol approvals, registrations and patients' consent}

The study was approved by the competent authority and registered at ClinicalTrials.gov under no NCT0179864. The local ethics committee approved the study. All patients gave their written informed consent.

\section{Clinical evaluation and experimental procedures}

All patients underwent a structured clinical evaluation that included the following rating scales: Unified Parkinson's Disease Rating Scale (UPDRS), Mini Nutritional Assessment (MNA) [20], modified Score of Emergency Geriatric Assessment (SEGAm) [21], and International Prostate Symptom Score (IPSS) [22]. Blood and lumbar CSF samples were collected for fluid biomarker assessment. Patients received an infusion test where the intracranial pressure (ICP) was recorded during a constant rate lumbar saline injection in order to characterize their cerebrospinal fluid (CSF) dynamics [18]. A majority of patients also underwent phase contrast (PC) magnetic resonance imaging (MRI). Combined with the ICP data, PCMRI data allowed a parameter characterizing the biomechanical response of the CNS, the CNS elastance coefficient, to be assessed, thus making this cohort unique for the purpose of our study. Finally, most patients underwent $\left[{ }^{123}\right.$ I]FP-CIT dopamine transporter (DAT) single-photon emission computed tomography (SPECT) imaging and FLAIR MRI.

\section{In vivo markers of neurodegenerative pathologies}

The presence of AD pathology was examined based on the ratio between CSF tau and amyloid beta $42\left(\mathrm{~A} \beta_{42}\right)$ peptides using a cut-off of 0.215 [23]. CSF levels of tau and $A \beta_{42}$ peptides were measured with standard commercial immunoassays. Presynaptic dopamine deficiency, which is a characteristic of Parkinsonian syndrome, was examined visually by an expert neurologist using $\left[{ }^{123}\right.$ I]FP-CIT DATSPECT. The presence of white matter lesions was ascertained on FLAIR MRI images using the Fazekas scale [24] with a cut-off of $\geq$ grade 2 . Finally, abnormal CSF dynamics was quantified via the CSF outflow resistance $\mathrm{R}_{0}$ derived from the lumbar infusion test [18] described in detail below. A threshold of $R_{0}>12 \mathrm{mmHg} / \mathrm{mL} / \mathrm{min}$ was chosen as a marker of altered CSF dynamics and increased risk of NPH. Although the above in vivo markers do not, by themselves, corroborate the clinical diagnosis of neurodegenerative diseases, they do flag an increased risk of having or developing them. For each marker, the prevalence of patients at risk was calculated only in patients with available data.

\section{Assessment of the biomechanical response of the CNS}

\section{PCMRI protocol and image analysis}

The 2D PCMRI was used as a non-invasive technique to quantify intracranial blood flows as reported elsewhere [25]. Patients who could not undergo PCMRI were excluded from the study. Other participants were imaged in a $3.0 \mathrm{~T}$ magnetic resonance (MR) scanner (Philips Achieva, Philips, Best, Netherlands) with a commercial 32-channel head coil. Retrospective cardiac-gated PCMRI sequences were performed with a plethysmograph set on the subject's finger to synchronize the acquired images with the subject's heartbeat. The selected segment was perpendicular to the intracranial internal carotids, basilar artery, straight sinus and sagittal sinus vessels. The acquisitions were made with an encoding velocity of $80 \mathrm{~cm} / \mathrm{s}$ and 32 time points were 
sampled for each cardiac cycle. Preprocessing steps, including velocity aliasing correction, noise reduction and vessel segmentation, were performed by a person blinded to the biomechanical data and using the dedicated Flow Analysis software, version 2018-12-12 [25]. Data identified by an independent reviewer as non-reliable due to poor image or segmentation quality were excluded from the study.

The instantaneous intracranial arterial flow $Q_{a}(t)$ was defined as the sum of blood flows in the intracranial internal carotid and basilar arteries. The instantaneous intracranial sinus flow $Q_{s}(t)$ was defined as the sum of blood flows in the straight and sagittal sinuses. It was assumed that during the whole cardiac cycle, the blood volume entering the cranial cavity through arteries was equal to the blood volume exiting it through veins. However, the straight and sagittal sinuses are not the only exit routes for blood. A correction factor was therefore defined to take the undetected blood volume exiting the cranial cavity into account: $\alpha_{\text {corr }}=\int_{0}^{T} Q_{a}(t) d t / \int_{0}^{T} Q_{s}(t) d t$, where $\mathrm{T}$ is the cardiac cycle period. The total instantaneous venous flow was then estimated as $Q_{v}(t)=\alpha_{\text {corr }} Q_{s}(t)$.

The instantaneous blood volume change was $V(t)=\int_{0}^{t} Q_{a}(t) d t-\int_{0}^{t} Q_{v}(t) d t$. The maximal blood volume change was definedas $\Delta V=\max \{V(t)\}_{t \in[0, T]}-\min \{V(t)\}_{t \in[0, T\}}$ Figure 1 shows a typical sample of PCMRI data and the associated arterial blood flow, venous blood flow and volume measurements.

\section{Infusion test protocol and intracranial pressure analysis}

Patients were awake and lying horizontally in the left lateral recumbent position. A lumbar puncture was performed with a needle connected to an infusion pump and to a pressure transducer (Fig. 2a). The CSF pressure, once zeroed at the third ventricle level, is a surrogate marker of ICP [26]. Fast ICP components are strongly coupled to the blood pressure and therefore have a pulsatile behaviour at the heart rate frequency. The ICP was digitized with a sampling frequency of $100 \mathrm{~Hz}$ and stored in a computer for offline analysis. First, the ICP was measured for $5 \mathrm{~min}$ at baseline, where the mean pressure was noted as $P_{b}$. Then, the lumbar subarachnoid space was infused with saline solution at a rate of $1.5 \mathrm{~mL} / \mathrm{min}$. Subsequently, ICP increased until a plateau was reached where the mean pressure was noted as $P_{\max }$. The resistance $R_{0}$ was calculated from the ICP mean curve as $R_{0}=\left(P_{\max }-\delta P-P_{b}\right) / I_{f}[18]$, where $\delta P$ is an artefact due to the needle resistance, which was easily measured on the ICP curve (Fig. 2b). For patients with elevated $R_{0}$, the test was sometimes stopped if ICP came close to the safety threshold of $40 \mathrm{mmHg}$ even if the plateau had not been reached. In this case, $P_{\max }$ was extrapolated from a sigmoid curve fit prediction.

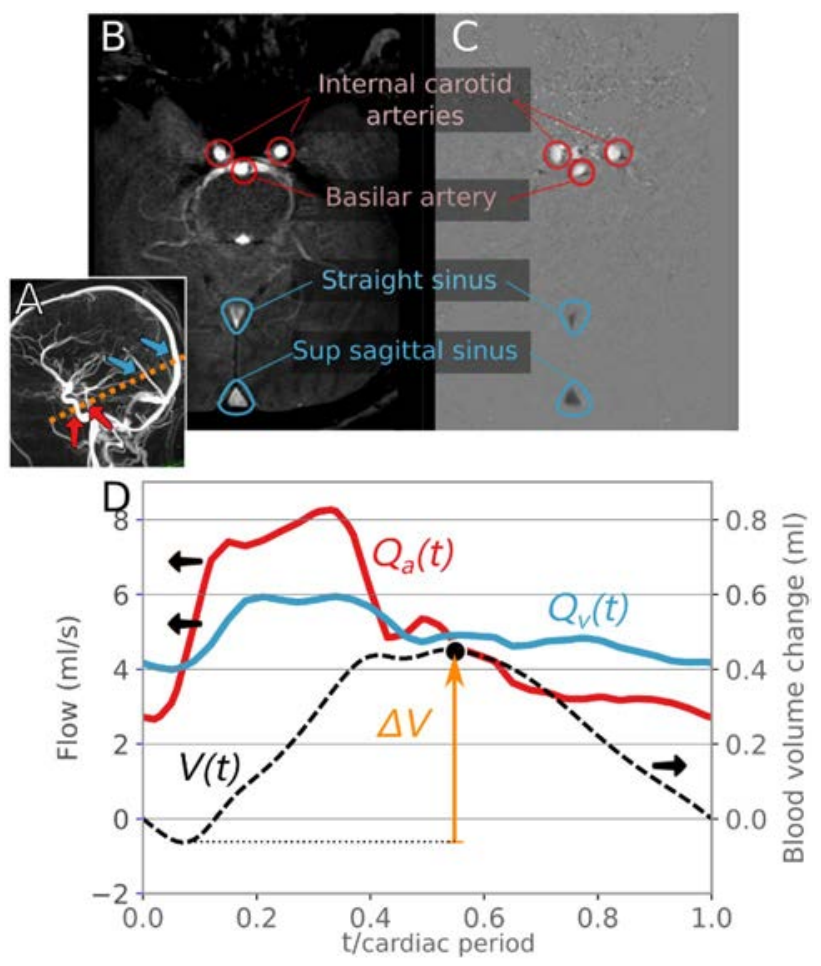

Fig. 1 Phase contrast MRI, typical blood flow measurements and analysis. Panel A: indication of the position of the 2D PC section on angiogram. Panel B: phase contrast angiogram used to determine acquisition location and level. Panel C: velocity-encoded MR images of the internal carotid arteries, basilar artery, straight sinus and superior sagittal sinus. Panel D: blood arterial flow $Q_{a}(t)$ (red curve), venous flow $Q_{v}(t)$ (blue curve), blood volume change $V(t)$ (black dashed curve) and maximal blood volume change $\Delta V$ (orange arrow) extracted from the PCMRI data. The left axis shows flow values; the right axis shows volume values

\section{CNS elastance coefficient assessment}

At each cardiac cycle, the pulsating blood flow induces a blood volume change $\Delta V$ and an ICP change $\Delta P$. Clinical observations showed that in a patient at rest with constant blood pressure and cardiac stroke volume, the change in pressure $\Delta P$ was proportional to the mean ICP [27]. The mathematical model proposed by Marmarou [28] provides the corresponding linear relationship $\Delta P=[\exp (E . \Delta V)-1]\left[P_{d}-\delta P-P_{0}\right]$, where $\mathrm{E}$ is the CNS elastance coefficient $(1 / \mathrm{mL}), P_{d}$ is the local diastolic pressure, and $P_{0}$ is the reference pressure $(\mathrm{mmHg})$. The CNS elastance coefficient characterizes the biomechanical response of the CNS and measures its ability to adapt to a change of volume (see "Discussion").

From the infusion test data and at each cardiac cycle, the ICP amplitude, $\Delta P$, was measured as the difference between the local maximum systolic pressure and local minimum diastolic pressure, $P_{d}$. A linear regression of $\Delta P$ versus $P_{d}$ dataset was performed to assess the slope 
Fig. 2 Lumbar infusion test, typical intracranial pressure measurements and analysis. Panel A: lumbar infusion test scheme: The intracranial pressure (ICP) is measured while a saline fluid is injected at a constant flow rate through a lumbar puncture. Panel B: ICP evolution during the infusion test. Blue line: ICP measurement; orange line: smoothed ICP (low-pass filter). The mean pressure at plateau, $P_{\max }$, and the mean pressure at baseline, $P_{\mathrm{b}}$, are indicated in black dotted lines. The pressure artefact induced by the needle, $\delta P$, the ICP amplitude, $\Delta P$, and the diastolic pressure, $P_{d}$, are indicated in red. Panel C: ICP amplitude, $\Delta P$, versus the diastolic pressure, $P_{d}$. Circles: measured values; black dashed line: linear regression. The reference pressure, $P_{0}$, and the slope, $\alpha$, are indicated in red

\section{A Lumbar infusion test}

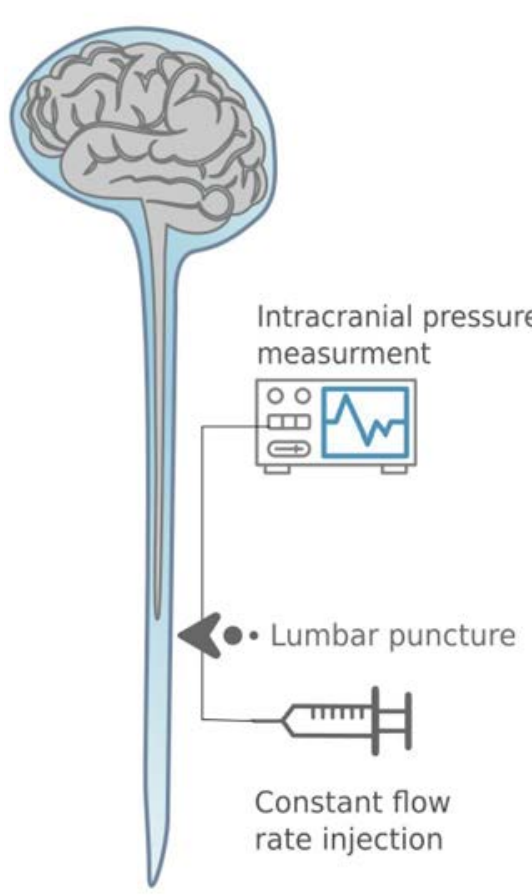

B Intracranial pressure (ICP)

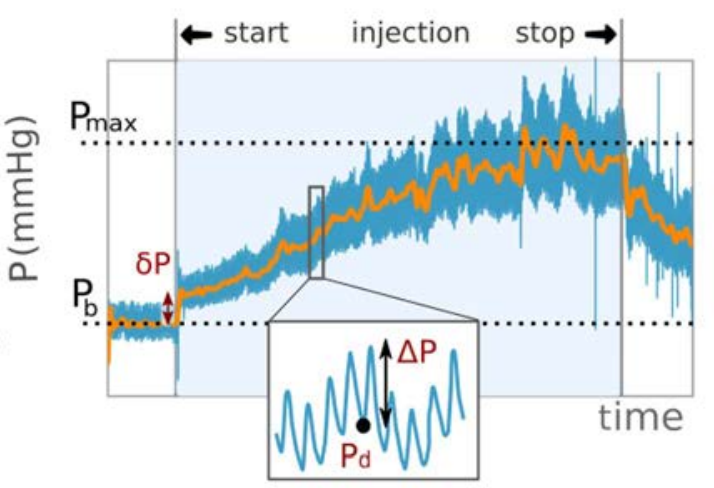

CICP pulse amplitude

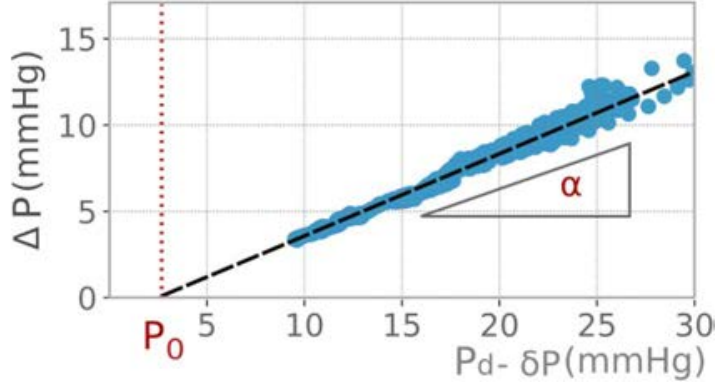

$\alpha=[\exp (E . \Delta V)-1]$ and the abscissa axis intersect $P_{0}$ (Fig. 2c). The regression used the random sample consensus algorithm [29], which gave robust regression, insensitive to the outliers induced by measurement artefacts or patients' movements. Fits with an R2 score lower than 0.9 were considered as non-reliable measures. The CNS elastance coefficient $E=\ln (\alpha+1) / \Delta V$ was deduced from the slope, $\alpha$, of the linear fit and from the blood volume change, $\Delta V$, previously calculated from PCMRI data.

\section{Frailty assessment}

Frailty was quantified by means of a frailty index (FI) based on the deficit accumulation approach, which has been widely validated across many settings and populations [30, 31]. The FI was defined as the ratio, ranging from 0 (not frail) to 1 (very frail), between the number of health deficits presented by the individual and the total number of deficits considered. A total of 40 variables were selected to measure the deficits in (1) executive functioning, (2) mentation, behaviour and mood, (3) gait, (4) rigidity and agility of extremities, (5) blood laboratory tests of markers related to kidney function, metabolic function and inflammatory conditions, which are of particular importance in older adults [32], (6) urinary symptoms, (7) nutrition and (8) history including medication intake and the presence of previously diagnosed hypertension or diabetes mellitus. Table 1 presents the variables and thresholds for deficit status. Following the guidelines of Searle et al. [30] for creating a frailty index, we did not include variables that had more than 5\% missing data and less than $1 \%$ of participants meeting criteria for the deficit. Patients presenting more than $10 \%$ of missing data on the 40 health deficits considered were excluded.

\section{Statistical analysis}

Statistical analyses were performed using STATA version 15 (College Station, TX). Descriptive analysis of the frequency distribution of all variables was performed. To ascertain normality, continuous variables were inspected through histograms and Skewness-Kurtosis tests. Depending on the distribution of the variables, the variable characteristics were presented as mean \pm SD or median with interquartile ranges (IQRs), the group differences were tested with a t-test or Wilcoxon rank-sum test and correlations were assessed by Pearson analysis or Spearman's rho analysis. The association between FI, age and the CNS elastance coefficient was further determined with a linear regression analysis. Three models were tested: one model (A) including age adjusted for sex, one model (B) where the CNS elastance coefficient was added, and a final model (C) where the CSF resistance to outflow $R_{0}$ was added as it seemed to be the principal covariate in a population suspected of NPH. For each model, regression coefficients, normalized effects 
Table 1 Variables used in the construction of the frailty index

\begin{tabular}{|c|c|c|c|c|}
\hline No & Variable & Condition for deficit & Deficits $(\%)$ & $\begin{array}{l}\text { Missing } \\
\text { data (\%) }\end{array}$ \\
\hline \multicolumn{5}{|c|}{ Executive functions } \\
\hline 1 & Dependence for dressing & UPDRS $^{\mathrm{a}}$ item score $\geq 2$ & 35 & 0 \\
\hline 2 & Dependence for hygiene & UPDRS item score $\geq 2$ & 35 & 0 \\
\hline 3 & Handwriting & UPDRS item score $\geq 2$ & 37 & 0 \\
\hline 4 & Speech & UPDRS item score $\geq 2$ & 12 & 0 \\
\hline 5 & Transferring (into and out of bed or chair) & UPDRS item score $\geq 2$ & 62 & 0 \\
\hline 6 & Chewing and swallowing & UPDRS item score $\geq 2$ & 7 & 0 \\
\hline 7 & Doing hobbies and other activities & UPDRS item score $\geq 2$ & 50 & 0 \\
\hline \multicolumn{5}{|c|}{ Mood and cognition } \\
\hline 8 & Cognitive impairment & UPDRS item score $\geq 2$ & 47 & 0 \\
\hline 9 & Depressed mood & SEGA item $>1$ & 7 & 0 \\
\hline 10 & Apathy & UPDRS item score $\geq 2$ & 93 & 0 \\
\hline 11 & Self-reported health compared to others & SEGA item $>1$ & 42 & 0 \\
\hline 12 & Night-time sleep problems & UPDRS item score $\geq 2$ & 23 & 0 \\
\hline 13 & Anxious mood & UPDRS item score $\geq 2$ & 30 & 0 \\
\hline \multicolumn{5}{|l|}{ Gait } \\
\hline 14 & Walking and balance & UPDRS item score $\geq 2$ & 65 & 0 \\
\hline 15 & Freezing & UPDRS item score $\geq 2$ & 23 & 0 \\
\hline 16 & Gait & UPDRS item score $\geq 2$ & 83 & 0 \\
\hline 17 & Global spontaneity of movement & UPDRS item score $\geq 2$ & 17 & 0 \\
\hline \multicolumn{5}{|c|}{ Rigidity and agility of extremities } \\
\hline 18 & Tapping fingers & $\begin{array}{l}\text { UPDRS item score } \\
\text { left }+ \text { right }>2\end{array}$ & 13 & 0 \\
\hline 19 & Tapping toes & $\begin{array}{l}\text { UPDRS item score } \\
\text { left }+ \text { right }>2\end{array}$ & 32 & 0 \\
\hline 20 & Rigidity upper extremities & $\begin{array}{l}\text { UPDRS item score } \\
\text { left }+ \text { right }>2\end{array}$ & 17 & 0 \\
\hline 21 & Rigidity lower extremities & $\begin{array}{l}\text { UPDRS item score } \\
\text { left }+ \text { right }>2\end{array}$ & 10 & 0 \\
\hline 22 & Hand movements & $\begin{array}{l}\text { UPDRS item score } \\
\text { left }+ \text { right }>2\end{array}$ & 17 & 0 \\
\hline 23 & Leg agility & $\begin{array}{l}\text { UPDRS item score } \\
\text { left }+ \text { right }>2\end{array}$ & 23 & 0 \\
\hline \multicolumn{5}{|c|}{ Laboratory blood test } \\
\hline 24 & Urea blood level & $<2.9 \mathrm{mmol} / \mathrm{L}$ or $>8.2 \mathrm{mmol} / \mathrm{L}$ & 17 & 0 \\
\hline 25 & Creatinine blood level & $\begin{array}{l}\text { Cockroft-Gault for- } \\
\text { mula }<60 \mathrm{~mL} / \mathrm{min} / 1.73 \mathrm{~m}^{2}\end{array}$ & 32 & 2 \\
\hline 26 & C-reactive protein blood level & $>3 \mathrm{mg} / \mathrm{L}$ & 32 & 0 \\
\hline 27 & Triglyceride blood level & $>2.25 \mathrm{mmol} / \mathrm{L}$ & 8 & 0 \\
\hline 28 & Glycated haemoglobin & $>5.7 \%$ & 62 & 5 \\
\hline 29 & Cobalamin & $<300 \mathrm{pg} / \mathrm{mL}$ & 28 & 5 \\
\hline 30 & Sodium & $<136 \mathrm{mmol} / \mathrm{L}$ or $>142 \mathrm{mmol} / \mathrm{L}$ & 13 & 0 \\
\hline \multicolumn{5}{|c|}{ Urinary symptoms } \\
\hline 31 & Need to urinate every $2 \mathrm{~h}$ & IPSS item $\geq 2$ & 35 & 0 \\
\hline 32 & Nocturia & IPSS item $\geq 2$ & 15 & 0 \\
\hline 33 & Leakage & IPSS item $\geq 2$ & 57 & 0 \\
\hline 34 & Sensation of bladder not empty & IPSS item $\geq 2$ & 15 & 0 \\
\hline \multicolumn{5}{|c|}{ Nutrition } \\
\hline 35 & Body mass index & $<18.5$ or $>25$ & 65 & 2 \\
\hline 36 & Loss of appetite & $\mathrm{MNA} \leq 2$ & 10 & 2 \\
\hline 37 & Loss of weight & $\mathrm{MNA} \leq 2$ & 10 & 2 \\
\hline
\end{tabular}


Table 1 (continued)

\begin{tabular}{|c|c|c|c|c|}
\hline No & Variable & Condition for deficit & Deficits (\%) & $\begin{array}{l}\text { Missing } \\
\text { data (\%) }\end{array}$ \\
\hline \multicolumn{5}{|c|}{ History } \\
\hline 38 & Drugs history & More than four drugs & 55 & 0 \\
\hline 39 & Hypertension & Present & 43 & 0 \\
\hline 40 & Diabetes mellitus & Present & 27 & 0 \\
\hline
\end{tabular}

a UPDRS Unified Parkinson's Disease Rating Scale

${ }^{\mathrm{b}}$ SEGA Score of Emergency Geriatric Assessment

${ }^{c}$ IPSS International Prostate Score Symptom

${ }^{\mathrm{d}}$ MNA Mini Nutritional Assessment

(beta) and p-values were reported. Skewness and kurtosis of standardized residuals were examined to ensure that assumptions of homoscedasticity were satisfied.

\section{Data availability}

Any qualified investigator may contact the principal investigator (E. S.) with details about (1) his qualifications, (2) the resources requested and (3) the proposed research question. All requests will be reviewed for availability and scientific merit.

\section{Results}

\section{Characteristics of the study population}

After careful evaluation, 40 patients were excluded from the initial cohort of 100 patients, for various reasons: missing data or low-quality PCMRI $(n=26)$, failure of the linear fit of $\Delta P$ versus $P_{d}$ dataset $(n=9)$ to fulfil the quality requirements $(\mathrm{R} 2>0.9)$, or more than $10 \%$ missing values in the FI $(n=5)$. This resulted in a final analytical sample of 60 patients, with statistical characteristics of demographic variables, frailty and biomechanical parameters as shown in Table 2.
Table 2 Demographics and characteristics of variables

\begin{tabular}{|c|c|c|c|c|}
\hline & Study sample & $\begin{array}{l}\text { Patients with CSF } \\
\text { dynamics disorders }^{\mathrm{a}}\end{array}$ & $\begin{array}{l}\text { Patients without CSF } \\
\text { dynamics disorders }\end{array}$ & $p$ value $^{\mathrm{c}}$ \\
\hline Sample size & 60 & 34 & 26 & - \\
\hline \multicolumn{5}{|l|}{ Demographics } \\
\hline Age (y) & $74[71,78]$ & $74[71,79]$ & $74[73,77]$ & 0.41 \\
\hline Female, $n(\%)$ & 50 & 50 & 50 & 0.89 \\
\hline \multicolumn{5}{|l|}{ Frailty } \\
\hline FI & $0.33 \pm 0.15$ & $0.34 \pm 0.15$ & $0.31 \pm 0.14$ & 0.57 \\
\hline \multicolumn{5}{|l|}{ Biomechanics } \\
\hline $\begin{array}{l}\text { Elastance coef. (/mL) } \\
\text { PCMRI characteristics }\end{array}$ & $0.52 \pm 0.13$ & $0.52 \pm 0.14$ & $0.51 \pm 0.12$ & 0.69 \\
\hline$\Delta V(\mathrm{~mL})$ & $0.74[0.58,0.99]$ & $0.68[0.56,0.94]$ & $0.78[0.6,1.1]$ & 0.21 \\
\hline \multicolumn{5}{|l|}{ ICP characteristics } \\
\hline Baseline pressure $(\mathrm{mmHg})$ & $10[7.6,11]$ & $10[9.2,12]$ & $8.7[6.9,10]$ & $0.02 *$ \\
\hline$P_{\max }(\mathrm{mmHg})$ & $30[23,35]$ & $34[31,42]$ & $22[19,26]$ & $<0.001^{*}$ \\
\hline$R_{0}(\mathrm{mmHg} / \mathrm{mL} / \mathrm{min})$ & $13[9.2,16]$ & $16[14,20]$ & $8.6[7.4,10]$ & $<0.001^{*}$ \\
\hline$P_{0}(\mathrm{mmHg})$ & $4.2 \pm 3.2$ & $4.3 \pm 3.1$ & $4 \pm 3.2$ & 0.84 \\
\hline$\alpha$ & $0.46[0.36,0.56]$ & $0.44[0.36,0.55]$ & $0.48[0.38,0.6]$ & 0.29 \\
\hline
\end{tabular}

* Results are considered as significant for $p<0.05$

${ }^{a}$ Patients having a CSF outflow resistance $>12 \mathrm{mmHg} / \mathrm{mL} / \mathrm{min}$

${ }^{\mathrm{b}}$ Patients having a CSF outflow resistance $\leq 12 \mathrm{mmHg} / \mathrm{mL} / \mathrm{min}$

${ }^{c} p$ value of the test to reject the null hypothesis that the difference in dynamics alteration between samples with and without CSF dynamics disorders is due to random sampling 
Their ages varied from 52 to 92 years with a median of 74 years (IQR 71-78). Concerning markers of neurodegenerative pathologies, $57 \%$ of patients had abnormal CSF dynamics and increased risk of NPH, 75\% had increased risk of $\mathrm{AD}, 25 \%$ (of 53 patients) had increased risk of Parkinsonian syndrome, and $62 \%$ (of 59 patients) had increased risk of vascular dementia. Also, among 52 patients without missing markers, $4 \%$ presented no risks of any of the four diseases considered, $19 \%$ presented risks of one disease, $38 \%$ presented risks of two diseases, $35 \%$ presented risks of three diseases, and $4 \%$ presented risks of the four diseases. Regarding vascular risk factors, $43 \%$ of patients were hypertensive and $27 \%$ were diabetic. Non-response analysis showed that the patients of the study sample were not significantly different from the excluded patients, concerning age, sex and prevalence of neurological disorders.

The blood volume change, $\Delta V$, measured from PCMRI, varied from 0.3 to $1.8 \mathrm{~mL}$ with a median of $0.74 \mathrm{~mL}$ (IQR $0.58-0.99)$. From ICP measurements, the fit slope, $\alpha$, varied from 0.23 to 1.2 with a median of 0.46 (IQR $0.36-0.56$ ) and the reference pressure, $P_{0}$, varied from -2 to $11 \mathrm{mmHg}$ with a mean value of $4.2 \pm 3.2 \mathrm{mmHg}$. The CNS elastance coefficient varied from 0.23 to $0.82 / \mathrm{mL}$ with a mean value of $0.52 \pm 0.13 / \mathrm{mL}$.

The FI had a right-skewed distribution (skewness value of 0.26 ) that could be approximated by a gamma distribution with a mean value of $0.33 \pm 0.15$, a minimum value of 0.075 and a maximum value of 0.62 . Table 1 shows the number of deficits and the percentage of missing data for each variable used to calculate the FI.

\section{Frailty, CNS elastance coefficient and ageing}

FI was significantly associated with age (rho $=0.38$, $p=0.003$ ). The CNS elastance coefficient was significantly correlated with FI $(r=0.32, p=0.014)$, but was independent

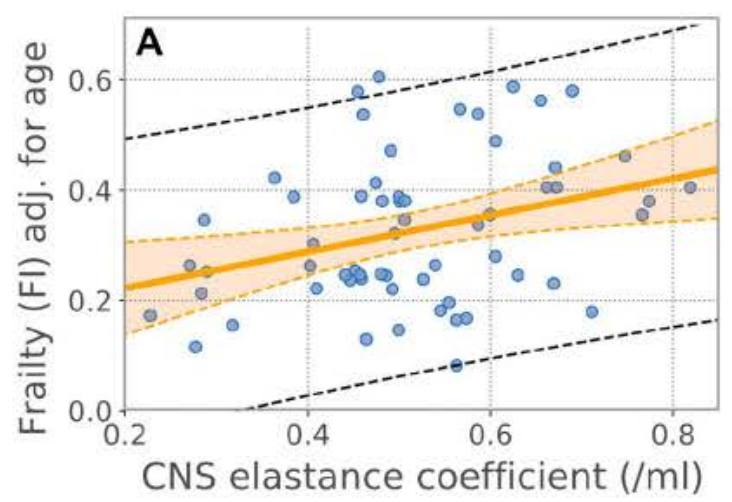

Fig. 3 CNS elastance coefficient and age relationships to frailty. Scatter plot of FI adjusted for age versus the CNS elastance coefficient (a) and scatter plot of FI adjusted for the CNS elastance coefficient of age (rho $=0.067, p=0.61)$. Thus, the age and the CNS elastance coefficient appeared to be two independent determinants of frailty. Figure 3 illustrates the relationship between FI, the CNS elastance coefficient and age.

A first linear regression model of FI including only age and sex (model A) explained 15\% of the FI variance. Sex had no significant effect on frailty in the population considered. When the CNS elastance coefficient was added (model B), the explained FI variance increased to $23 \%$. In this model, both the CNS elastance coefficient and age were associated with frailty $(p<0.05)$ with standardized beta coefficients of 0.3 and 0.36 , respectively. Table 3 presents details on the regression models.

\section{Frailty components, ageing and CNS elastance coefficient}

To investigate whether there were specific health deficits associated with an increased CNS elastance coefficient or age, we analysed the mean differences in value between the sample with deficit and the sample without deficit for each FI variable. The relative mean differences are plotted on the chart of Fig. 4. The CNS elastance coefficient was higher in the group of patients with deficit for $95 \%$ of the FI variables, but the difference was only statistically significant for the two variables of global movement spontaneity and medication intake. Similarly, the age was higher in patients having a deficit for $78 \%$ of the FI variables and every age difference was statistically significant.

\section{Effects of CSF dynamics disorders}

The CSF outflow resistance $R_{0}$ varied from $6.2 \mathrm{mmHg} /$ $\mathrm{mL} / \mathrm{min}$ to $42 \mathrm{mmHg} / \mathrm{mL} / \mathrm{min}$ with a median of $13 \mathrm{mmHg} /$ $\mathrm{mL} / \mathrm{min}$ (IQR 9.2-16). Table 2 compares the variables between groups of patients with and without elevated $R_{0}$.

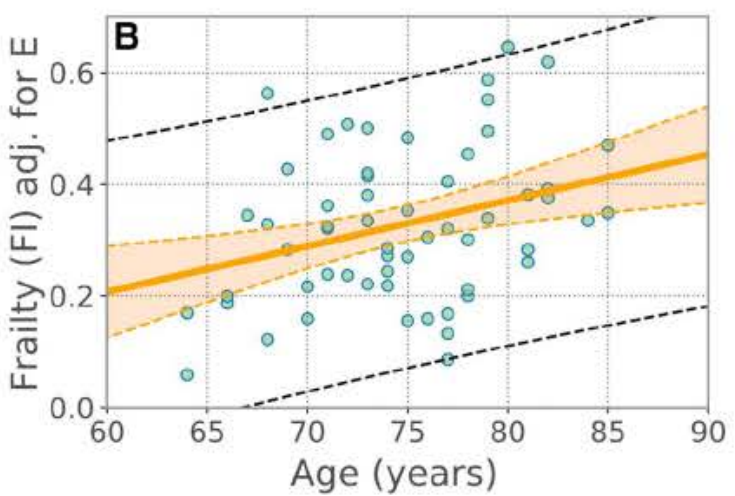

versus the age (b). The linear regression (orange lines) with $95 \%$ confidence bands (orange dashed lines) and 95\% prediction bands (black dashed lines) are indicated 
Table 3 Regression analysis of frailty

\begin{tabular}{|c|c|c|c|c|c|c|c|c|c|}
\hline$\overline{\text { FI }}$ & Model A & & & Model B & & & Model C & & \\
\hline$N$ & 60 & & & 60 & & & 60 & & \\
\hline$R$-squared (\%) & 15 & & & 23 & & & 24 & & \\
\hline Variables & Coef. & Beta & $P>|t|$ & Coef. & Beta & $P>|t|$ & Coef. & Beta & $P>|t|$ \\
\hline Elastance coef. $(/ \mathrm{mL})$ & - & - & - & $0.33 *$ & $0.3^{*}$ & 0.012* & $0.33 *$ & $0.3^{*}$ & $0.012 *$ \\
\hline Age (y) & $0.0084 *$ & $0.37 *$ & $<0.01 *$ & $0.0082 *$ & $0.36^{*}$ & $<0.01^{*}$ & $0.0083 *$ & $0.36 *$ & $<0.01 *$ \\
\hline Sex & -0.013 & -0.046 & 0.71 & -0.014 & -0.05 & 0.68 & -0.014 & -0.048 & 0.68 \\
\hline$R_{0}(\mathrm{mmHg} / \mathrm{mL} / \mathrm{min})$ & - & - & - & - & - & - & -0.002 & -0.093 & 0.43 \\
\hline
\end{tabular}

*Results are considered as significant for $p<0.05$

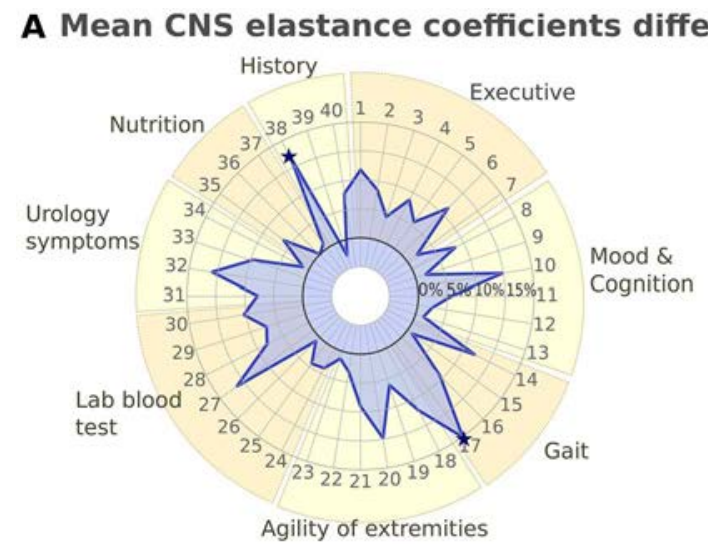

Fig. 4 CNS elastance coefficient and age relationships to the components of frailty. The relative difference of mean CNS elastance coefficients (a) and mean ages (b) in the samples with and without deficit

The baseline pressure was $15 \%$ higher $(p=0.02)$ and the maximum pressure $P_{\max }$ during the infusion test was $54 \%$ higher $(p<0.001)$ in the group of patients with elevated CSF outflow resistance $\left(R_{0}>12 \mathrm{mmHg} / \mathrm{mL} / \mathrm{min}\right)$. No significant differences appear on the other variables between the two groups. The linear regression was not much affected by adding the CSF outflow resistance to the model (Table 3, model C).

\section{Discussion}

We found a significant association between the CNS elastance coefficient and frailty in a cohort of older adults suspected of NPH and presenting multiple comorbid neuropathological conditions. This association was maintained even after adjustment for CSF outflow resistance, showing that it was not primarily driven by CSF dynamics abnormalities that are characteristic of NPH. Our findings provide the first evidence that altered biomechanical response of the

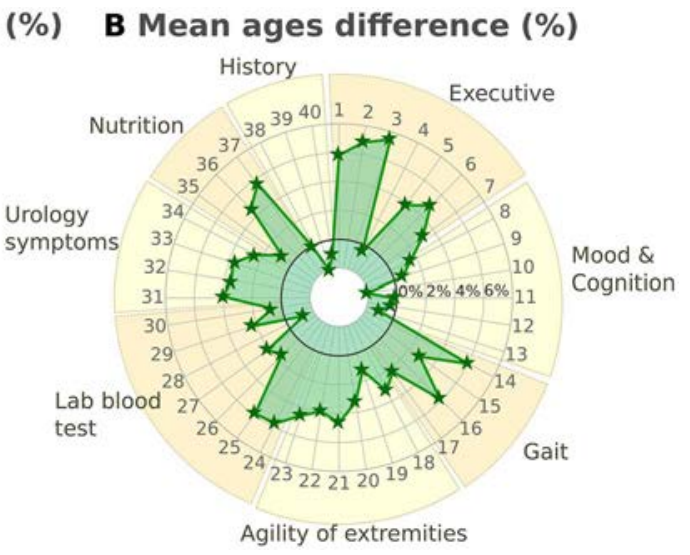

are indicated for each FI variable as labelled in Table 1. Significant differences $(p<0.05)$ are indicated with stars.

CNS is associated with global health deficits in older adults undergoing pathological brain ageing.

Neurodegenerative diseases are usually characterized according to their distinct clinical and pathological features. However, recent evidence suggests that age-related neurodegenerative diseases are often accompanied by multiple, partly overlapping, pathological brain changes [33, 34]. In this context, the association found here between the CNS elastance coefficient and the FI can be interpreted as a characteristic of pathological ageing of the brain, which is not specific to any neurodegenerative disease in particular, for four following reasons. (1) Although the patients of our study were suspected of NPH, not all presented CSF dynamic alterations (43\% had normal CSF outflow resistance and were not shunted). (2) The regression model of frailty remained unchanged when the CSF outflow resistance was considered. Neither the frailty nor the CNS elastance coefficient differed between patients with or without increased CSF resistance to outflow. Therefore, the results were not specific to altered CSF dynamics. This suggests 
that they were not specific to NPH and makes the absence of a non-NPH control group acceptable. (3) The CNS elastance coefficient depends on the brain structure and the CNS tissue elasticity [35], both of which are modified in various neurodegenerative diseases [36-39]. (4) Patients suspected of NPH often present neurodegenerative comorbidities [17]. Typical AD histological markers have been observed in $42 \%$ of NPH patient brain biopsies [40]. Vascular risk factors such as hypertension and diabetes mellitus as well as white matter lesions were related to clinical and imaging features of NPH [41]. Also, the triad of clinical findings classic in NPH (gait deviation, cognitive impairments and urinary incontinence) commonly occurs in other neurodegenerative diseases and non-specifically in advanced age [16]. Our results support these observations: half the patients presented vascular risk factors, $96 \%$ presented clinical and neurobiological markers known to be associated with NPH, AD, Parkinsonian syndrome or vascular dementia, and $77 \%$ had risks of more than two of these neurodegenerative diseases. So, the present cohort is a convenient model of pathological brain ageing with a wide range of degrees of frailty (FI varying from 0.075 to 0.62 ), suitable to assess the relationship between frailty and the biomechanical response of the CNS in a non-specific disease context.

A wealth of literature supports the idea that frailty is associated with pathological brain ageing. For example, accelerated progression of frailty was associated with subclinical levels of AD or Lewy body pathology, nigral neuronal loss and macroinfarcts in community-dwelling older adults [12]. Previous research has also observed that frail individuals are more likely to have clinically diagnosed dementia, including $\mathrm{AD}$, vascular dementia and all dementia among communitydwelling older people [4-6]. Taken together, these findings suggest that the association of frailty with neurodegenerative diseases (1) is not specific to any brain pathology in particular, (2) may be present years before the onset of overt clinical symptoms and (3) can represent a systemic vulnerability. This is consistent with the concept of brain ageing as an accumulation of brain alterations, progressing insidiously before clinical symptoms appear [1], at a rate that depends on the complex interplay between regulation mechanisms and stressors, e.g. biological, biochemical or biomechanical stressors. In pathological ageing, an increased vulnerability to stressors accelerates this accumulation, leading to an earlier manifestation of clinical symptoms (see Fig. 5). The frailty concept allows this multi-organ vulnerability [42] to be measured.

In particular, the CNS elastance coefficient can be interpreted as a measure of the vulnerability to mechanical stressors of the brain organ. This coefficient modulates the relative ICP changes in response to intracranial volume changes. It is usually estimated by measuring the ICP response following a controlled, fast volume injection into the subarachnoid

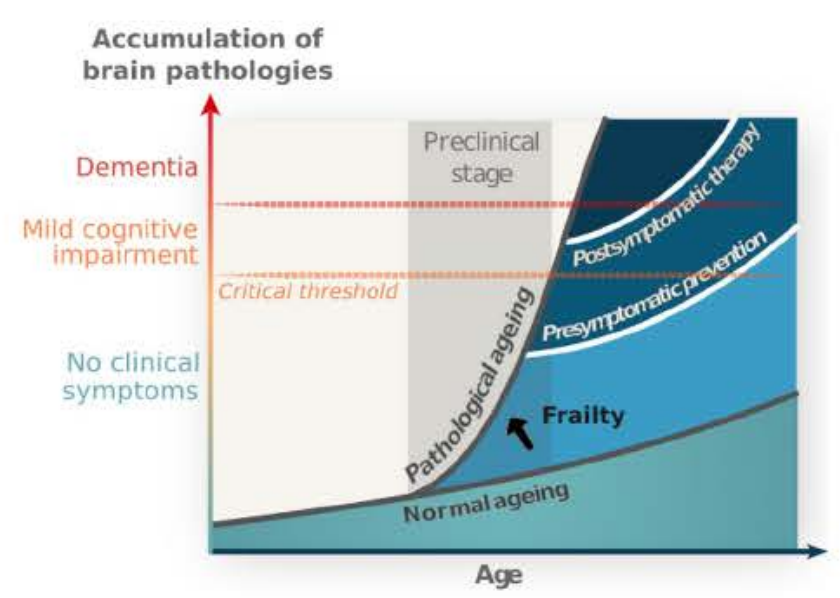

Fig. 5 Normal and pathological brain ageing, a conceptual view. Brain ageing is conceptualized as an accumulation of brain pathologies that progress insidiously until clinical symptoms appear. Frailty increases vulnerability to stressors and leads to pathological, accelerated, brain ageing with an earlier crossing of the critical threshold of clinical symptoms manifestation

or ventricular space, i.e. an artificial mechanical stressor. Since PCMRI and lumbar infusion test were performed in all patients of our cohort, we were able, for the first time, to estimate the CNS elastance coefficient from the blood pulsatility, i.e. a natural mechanical stressor. A CNS with low elastance coefficient buffers the relative ICP change in response to an intracranial volume perturbation and, conversely, a higher elastance coefficient yields an increased ICP change in response to the same volume perturbation (see Fig. 6). Thus, an increased CNS elastance coefficient leads to a higher risk of excessive ICP peaks, which are known to induce brain damage [43]. Interestingly, in our regression model, the strength of the effect of the CNS elastance coefficient on frailty was comparable to that of age, which is known to be the main risk factor for frailty [3]. This strong positive correlation between frailty and CNS elastance coefficient means that a frail person is likely to have abnormal ICP peaks, making the brain more vulnerable. This mechanism may contribute to the link between frailty and neurodegenerative diseases.

Both alterations of the brain CNS elastance coefficient and neurodegenerative diseases appear to result from an accumulation of health deficits. Frailty is indeed more accurate in the prediction of dementia than single risk factors taken separately [5]. Even health deficits that may seem irrelevant to cognitive impairment have been shown to be associated with dementia when combined in an FI [6]. In the same way, our results show that the CNS elastance coefficient is not significantly different between patients with or without a given specific health deficit for almost all the FI variables, while being significantly correlated to the global FI. The deficit accumulation approach of the FI translates 


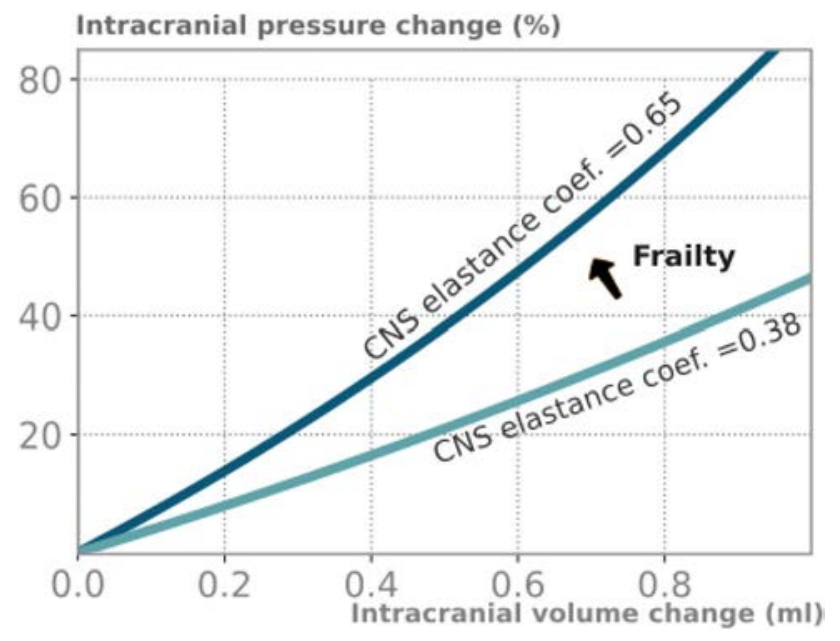

Fig. 6 Higher CNS elastance coefficient increases vulnerability to mechanical stressors. The intracranial pressure (ICP) relative change, $\Delta P / P$, induced by an instantaneous intracranial volume change $\Delta V$ is plotted for a low and a high value of the CNS elastance coefficient (study mean value \pm standard deviation). The prediction is made according to Marmarou's model, $\Delta P=[\exp (E . \Delta V)-1]\left[P-P_{0}\right]$, where $P_{0}$ is neglected. This study shows that greater frailty is associated with higher CNS elastance coefficient, leading to an increased risk for excessive ICP peaks, i.e. to an increased brain vulnerability to mechanical stressors

the idea that factors weakly associated with neurodegenerative disorders can, when taken together, still contribute to a global condition leading to neurodegenerative diseases. This suggests that the CNS biomechanical alterations, associated with general health deficits, might set up conditions of increased vulnerability that lead to the onset and progression of neurodegenerative diseases.

Given that age-related neurodegenerative diseases are often characterized by multiple brain alterations combining lesions of several, partly overlapping, neuropathologies [44], it is essential to capture multi-factorial causes. It has recently been suggested that dynamic indicators of the response of physiological systems to stimuli can add value to the static FI measurements of health deficits [45]. The dynamic approach indeed gives additional information on the systemic resilience and the recovery potential of a patient. Altogether, the above results suggest that the CNS elastance coefficient, being a quantitative indicator of the CNS response to blood pulsatility, could be a dynamic indicator that would improve frailty assessment. More generally, the CNS biomechanical characterization may be a relevant modality, in addition to conventional clinical and imaging techniques, to identify pathological changes in the brain. It could therefore help to identify the shift from normal to pathological brain ageing as recent studies have shown the benefits of multi-modal approaches for this purpose [46, 47].

This study had some limitations. First, our results relied on the assumption that the blood volume change $\Delta V$ was the same during the PCMRI examination and the infusion test, which were not performed at the same time. However, PCMRI flux measurements have been reported to be reproducible [48], indicating that the haemodynamics remains unchanged when patients are in similar conditions. Here, the patients were lying horizontally in both examinations, had small cardiac frequency differences between the two occasions $(6 \pm 5 \%)$, and were examined within a short time interval ( $3 \pm 6$ days). Second, the cross-sectional design of the study did not allow the directionality of the association between frailty and the CNS elastance coefficient to be resolved. Third, variables not considered in the present study may be responsible for the apparent relationship between frailty and CNS elastance coefficient. Noteworthy, we checked that the main confounder related to our NPHsuspected population, the resistance to CSF outflow (R0), is not such a confounder variable. Fourth, our study does not provide information on the relevance of our approach in the preclinical stage as all patients presented clinical symptoms. The absence of an age-matched control group did not allow exploring whether the CNS elastance coefficient can distinguish pathological from normal brain ageing. The invasiveness of the lumbar puncture limits such a wider applicability of our approach. A range of alternative non-invasive ICP monitoring techniques are emerging, including monitoring of the optic nerve sheath diameter or of the tympanic membrane displacement [49]. These techniques will surely be improved in the near future to eventually provide reliable quantification tools of biomechanical markers. This will allow the predictive value of CNS biomechanical markers for dementia to be explored using longitudinal designs in larger samples of community-dwelling older adults. Such studies are needed to resolve the directionality of the association between frailty and the CNS elastance coefficient and to explore the preclinical stage.

\section{Conclusions}

There is an urgent need to identify new quantitative markers of the shift from normal to pathological brain ageing. Preventive intervention strategies, including health-promoting life choices, are thought to be our best chance of avoiding or delaying the onset and development of neurodegenerative diseases. This study shows that the biomechanical response of the CNS is associated with the FI, which is known to predict dementia. By quantifying the CNS response to mechanical stressors such as the blood pulsatility, the biomechanical characterization of the CNS provides dynamic information that goes beyond the FI static measurement of health deficits. Hence, the CNS biomechanical characterization, as a complementary tool to the FI, may help the early detection 
of pathological ageing of the brain and should be further investigated in longitudinal studies.

Acknowledgements This study was supported by a grant from the French Ministry of Health with the participation of the Groupement Interrégional de Recherche Clinique et d'Innovation Sud-Ouest OutreMer Hospitalier (PHRCInterRégional 2010), by research funding from the Occitania Region (RPBIO 2015 no 14054344), and by the European Research Council under the European Union's Seventh Framework Program (FP7/2007-2013)/ERC grant agreement no 615102 (https:// erc.europa.eu/).

Author contribution AV designed and conceptualized the study, processed, analysed and interpreted the data, performed the statistical analysis, and drafted and revised the manuscript. NDC and EH designed and conceptualized the study on the frailty part, interpreted the data, and drafted and revised the manuscript. AL segmented and analysed PCMRI data. OB provided tools and methods for PCMRI acquisition and data processing and analysed PCMRI data. ZC interpreted ICP measurements and revised the manuscript. LB and PP played a major role in the acquisition of clinical data and MRI data, respectively. PS designed and conceptualized the biomechanical part of the study, interpreted the data, and drafted and revised the manuscript. SL and ES designed and conceptualized the study, interpreted the data, drafted and revised the manuscript, supervised the study and obtained funding.

\section{Compliance with ethical standards}

Conflicts of interest On behalf of all authors, the corresponding author states that there is no conflict of interest.

\section{References}

1. Jack CR, Knopman DS, Jagust WJ et al (2010) Hypothetical model of dynamic biomarkers of the Alzheimer's pathological cascade. Lancet Neurol 9:119-128. https://doi.org/10.1016/S1474 -4422(09)70299-6

2. Sperling RA, Karlawish J, Johnson KA (2013) Preclinical Alzheimer disease-the challenges ahead. Nat Rev Neurol 9:54-58. https ://doi.org/10.1038/nrneurol.2012.241

3. Cesari M, Vellas B, Gambassi G (2013) The stress of aging. Exp Gerontol 48:451-456. https://doi.org/10.1016/j.exger.2012.10.004

4. Kojima G, Taniguchi Y, Iliffe S, Walters K (2016) Frailty as a predictor of alzheimer disease, vascular dementia, and all dementia among community-dwelling older people: a systematic review and meta-analysis. J Am Med Dir Assoc 17:881-888. https://doi. org/10.1016/j.jamda.2016.05.013

5. Song X, Mitnitski A, Rockwood K (2014) Age-related deficit accumulation and the risk of late-life dementia. Alzheimers Res Ther 6:54. https://doi.org/10.1186/s13195-014-0054-5

6. Song X, Mitnitski A, Rockwood K (2011) Nontraditional risk factors combine to predict Alzheimer disease and dementia. Neurology 77:227-234. https://doi.org/10.1212/WNL.0b013e318225c6b

7. Oppenheim H, Paolillo EW, Moore RC et al (2018) Neurocognitive functioning predicts frailty index in HIV. Neurology 91:e162e170. https://doi.org/10.1212/WNL.0000000000005761

8. Robertson DA, Savva GM, Kenny RA (2013) Frailty and cognitive impairment-a review of the evidence and causal mechanisms. Ageing Res Rev 12:840-851. https://doi.org/10.1016/j. arr.2013.06.004
9. Rolfson DB, Wilcock G, Mitnitski A et al (2013) An assessment of neurocognitive speed in relation to frailty. Age Ageing 42:191196. https://doi.org/10.1093/ageing/afs185

10. Kant IMJ, de Bresser J, van Montfort SJT et al (2018) The association between brain volume, cortical brain infarcts, and physical frailty. Neurobiol Aging 70:247-253. https://doi.org/10.1016/j. neurobiolaging.2018.06.032

11. Gallucci M, Piovesan C, Di Battista ME (2018) Associations between the Frailty Index and Brain Atrophy: the Treviso Dementia (TREDEM) Registry. J Alzheimers Dis 62:1623-1634. https:// doi.org/10.3233/JAD-170938

12. Buchman AS, Yu L, Wilson RS et al (2013) Association of brain pathology with the progression of frailty in older adults. Neurology 80:2055-2061. https://doi.org/10.1212/WNL.0b013e3182 $94 \mathrm{~b} 462$

13. Goriely A, Geers MGD, Holzapfel GA et al (2015) Mechanics of the brain: perspectives, challenges, and opportunities. Biomech Model Mechanobiol 14:931-965. https://doi.org/10.1007/s1023 7-015-0662-4

14. Holter KE, Kehlet B, Devor A et al (2017) Interstitial solute transport in 3D reconstructed neuropil occurs by diffusion rather than bulk flow. Proc Natl Acad Sci 114:9894-9899. https://doi. org/10.1073/pnas.1706942114

15. Hernández JCC, Bracko O, Kersbergen CJ et al (2019) Neutrophil adhesion in brain capillaries reduces cortical blood flow and impairs memory function in Alzheimer's disease mouse models. Nat Neurosci 22:413. https://doi.org/10.1038/s41593-018-0329-4

16. Klassen BT, Ahlskog JE (2011) Normal pressure hydrocephalus. Neurology 77:1119. https://doi.org/10.1212/WNL.0b013e3182 $2 \mathrm{f02f5}$

17. Malm J, Graff-Radford NR, Ishikawa M, et al. (2013) Influence of comorbidities in idiopathic normal pressure hydrocephalus research and clinical care. A report of the ISHCSF task force on comorbidities in INPH. Fluids Barriers CNS 10:22. https://doi. org/10.1186/2045-8118-10-22**

18. Marmarou A, Young HF, Aygok GA et al (2005) Diagnosis and management of idiopathic normal-pressure hydrocephalus: a prospective study in 151 patients. J Neurosurg 102:987-997. https:// doi.org/10.3171/jns.2005.102.6.0987

19. Relkin N, Marmarou A, Klinge P et al (2005) Diagnosing idiopathic normal-pressure hydrocephalus. Neurosurgery. https://doi. org/10.1227/01.neu.0000168185.29659.c5

20. Vellas B, Guigoz Y, Garry PJ et al (1999) The mini nutritional assessment (MNA) and its use in grading the nutritional state of elderly patients. Nutrition 15:116-122. https://doi.org/10.1016/ S0899-9007(98)00171-3

21. Feck E, Zulfiqar AA (2018) Screening of frailty in family practice by the modified SEGA grid. Rev Med Liege 73:513-518

22. Araki I, Kuno S (2000) Assessment of voiding dysfunction in Parkinson's disease by the international prostate symptom score. J Neurol Neurosurg Psychiatry 68:429-433. https://doi. org/10.1136/jnnp.68.4.429

23. Mo Y, Stromswold J, Wilson K et al (2017) A multinational study distinguishing Alzheimer's and healthy patients using cerebrospinal fluid tau/A $\beta 42$ cutoff with concordance to amyloid positron emission tomography imaging. Alzheimers Dement Diagn Assess Dis Monit 6:201-209. https://doi.org/10.1016/j.dadm.2017.02.004

24. Fazekas F, Chawluk JB, Alavi A et al (1987) MR signal abnormalities at $1.5 \mathrm{~T}$ in Alzheimer's dementia and normal aging. AJR Am J Roentgenol 149:351-356. https://doi.org/10.2214/ajr.149.2.351

25. Balédent O, Henry-Feugeas M-C, Idy-Peretti I (2001) Cerebrospinal fluid dynamics and relation with blood flow: a magnetic resonance study with semiautomated cerebrospinal fluid segmentation. Invest Radiol 36:368

26. Lenfeldt N, Koskinen L-OD, Bergenheim AT et al (2007) CSF pressure assessed by lumbar puncture agrees with intracranial 
pressure. Neurology 68:155-158. https://doi.org/10.1212/01. wnl.0000250270.54587.71

27. Szewczykowski J, liwka S, Kunicki A et al (1977) A fast method of estimating the elastance of the intracranial system. J Neurosurg 47:19-26. https://doi.org/10.3171/jns.1977.47.1.0019

28. Marmarou A, Shulman K, Rosende RM (1978) A nonlinear analysis of the cerebrospinal fluid system and intracranial pressure dynamics. J Neurosurg 48:332-344. https://doi.org/10.3171/ jns.1978.48.3.0332

29. Fischler MA, Bolles RC (1981) Random sample consensus: a paradigm for model fitting with applications to image analysis and automated cartography. Commun ACM 24:381-395. https:// doi.org/10.1145/358669.358692

30. Searle SD, Mitnitski A, Gahbauer EA et al (2008) A standard procedure for creating a frailty index. BMC Geriatr 8:24. https:// doi.org/10.1186/1471-2318-8-24

31. Rockwood K, Mitnitski A (2011) Frailty defined by deficit accumulation and geriatric medicine defined by frailty. Clin Geriatr Med 27:17-26. https://doi.org/10.1016/j.cger.2010.08.008

32. Vásárhelyi B, Debreczeni LA (2017) Lab test findings in the elderly. EJIFCC 28:328-332

33. Irwin DJ, Grossman M, Weintraub D et al (2017) Neuropathological and genetic correlates of survival and dementia onset in synucleinopathies: a retrospective analysis. Lancet Neurol 16:55-65. https://doi.org/10.1016/S1474-4422(16)30291-5

34. Iturria-Medina Y, Sotero RC, Toussaint PJ et al (2016) Early role of vascular dysregulation on late-onset Alzheimer/'s disease based on multifactorial data-driven analysis. Nat Commun 7:11934. https://doi.org/10.1038/ncomms11934

35. Wirth B, Sobey I (2009) Analytic solution during an infusion test of the linear unsteady poroelastic equations in a spherically symmetric model of the brain. Math Med Biol J IMA 26:25-61. https ://doi.org/10.1093/imammb/dqn021

36. Pini L, Pievani M, Bocchetta M et al (2016) Brain atrophy in Alzheimer's Disease and aging. Ageing Res Rev 30:25-48. https ://doi.org/10.1016/j.arr.2016.01.002

37. Murphy MC, Huston J, Jack CR et al (2011) Decreased brain stiffness in Alzheimer's disease determined by magnetic resonance elastography. J Magn Reson Imaging JMRI 34:494-498. https:// doi.org/10.1002/jmri.22707

38. Sack I, Streitberger K-J, Krefting D et al (2011) The influence of physiological aging and atrophy on brain viscoelastic properties in humans. PLOS One 6:e23451. https://doi.org/10.1371/journ al.pone. 0023451

39. Streitberger K-J, Wiener E, Hoffmann J et al (2011) In vivo viscoelastic properties of the brain in normal pressure hydrocephalus. NMR Biomed 24:385-392. https://doi.org/10.1002/nbm.1602

40. Leinonen V, Koivisto AM, Alafuzoff I et al (2012) cortical brain biopsy in long-term prognostication of 468 patients with possible normal pressure hydrocephalus. Neurodegener Dis 10:166-169. https://doi.org/10.1159/000335155

41. Jaraj D, Agerskov S, Rabiei K et al (2016) Vascular factors in suspected normal pressure hydrocephalus. Neurology 86:592. https ://doi.org/10.1212/WNL.0000000000002369

42. Clegg A, Young J, Iliffe S et al (2013) Frailty in elderly people. Lancet 381:752-762. https://doi.org/10.1016/S0140 $-6736(12) 62167-9$

43. Stone J, Johnstone DM, Mitrofanis J, O'Rourke M (2015) The mechanical cause of age-related dementia (Alzheimer's disease): the brain is destroyed by the pulse. J Alzheimers Dis 44:355-373. https://doi.org/10.3233/JAD-141884

44. Rahimi J, Kovacs GG (2014) Prevalence of mixed pathologies in the aging brain. Alzheimer's Res Ther 6:82. https://doi. org/10.1186/s13195-014-0082-1

45. Olde Rikkert MGM, Melis RJF (2019) Rerouting geriatric medicine by complementing static frailty measures with dynamic resilience indicators of recovery potential. Front Physiol. https://doi. org/10.3389/fphys.2019.00723

46. Khan TK (2018) An algorithm for preclinical diagnosis of alzheimer's disease. Front Neurosci. https://doi.org/10.3389/fnins .2018 .00275

47. Payton NM, Kalpouzos G, Rizzuto D et al (2018) Combining cognitive, genetic, and structural neuroimaging markers to identify individuals with increased dementia risk. J Alzheimers Dis JAD 64:533-542. https://doi.org/10.3233/JAD-180199

48. Spilt A, Box FMA, van der Geest RJ et al (2002) Reproducibility of total cerebral blood flow measurements using phase contrast magnetic resonance imaging. J Magn Reson Imaging JMRI 16:15. https://doi.org/10.1002/jmri.10133

49. Khan MN, Shallwani H, Khan MU, Shamim MS (2017) Noninvasive monitoring intracranial pressure: a review of available modalities. Surg Neurol Int. https://doi.org/10.4103/sni.sni_403_16 